\title{
High-sensitivity and large-dynamic-range fiber refractometer based on composite-cavity Fabry-Perot structure
}

\author{
Pengcheng Chen ${ }^{1}$, Xuewen Shu ${ }^{1, *}$, Haoran Cao ${ }^{1}$, Kate Sugden ${ }^{2}$ \\ ${ }^{1}$ Wuhan National Laboratory for Optoelectronics \& School of Optoelectronic Science and \\ Engineering, Huazhong University of Science and Technology, Wuhan 430074, China \\ ${ }^{2}$ Aston Institute of Photonic Technologies, Aston University, Birmingham B4 7ET, UK \\ * Corresponding author: xshu@hust.edu.cn
}

\begin{abstract}
Most sensors have the tradeoff dilemma of high sensitivity and large dynamic range. We demonstrate here an all-fiber refractive index sensor based on a composite intrinsic Fabry-Perot interferometer (FPI), which possesses the co-existence advantages of high sensitivity and large dynamic range. Experimental trends are in good agreement with the theoretical predictions. The co-existence of high sensitivity and large dynamic range in a composite FPI is of great significance to practical refractive index measurement.
\end{abstract}

Keywords: Fabry-Perot, refractive index sensing, high sensitivity larger dynamic range co-existence.

\section{INTRODUCTION}

Sensors with both high sensitivity and large dynamic range are very desirable for many applications, however, most sensors face a tradeoff between the two. Refractive index (RI) sensors, which are important sensors in the fields of physics, chemistry, biomedicine and environmental science, also have such a dilemma. Most effort to date has been to improve the sensitivity of such sensors. However, for wavelength demodulated sensors such as micro/nanofiber devices [1], long-period fiber gratings [2], etc., their high RI sensitivities usually only apply to a very small RI range. Beyond the specified RI range, the sensing performance will be greatly reduced or even stop functioning. Intensity demodulated sensors include devices such as micro channel [3] through fiber core and various interferometers $[4,5]$. The sensitivities of those reported intensity demodulated schemes based on are relatively low, although a high sensitivity has recently been demonstrated with a Fabry-Perot interferometer created using a femtosecond laser (fs-laser) [6]. Indeed, RI sensors that can achieve both high sensitivity and large dynamic range have seldom been reported.

In this paper, we report that composite-cavity FPI based RI sensors have the potential to solve the tradeoff difficulty of high sensitivity and large dynamic range. Our investigation shows that different interference fringe dips of the composite FPI can correspond to a series of different ultra-sensitive zones for RI measurement. Therefore, high sensitivity and large dynamic range can co-exist in such structures. The trends shown in experiments are in good accord with the theoretical analysis and simulations.

\section{WORK PRINCIPLE}

Figure 1 shows the schematic diagram of the proposed composite FPI, which comprises of a well-cleaved SMF and a small refractive-index-modified-dot (RIMD) in the fiber core. Due to the refractive index difference, the composite cavity has three reflectors: SMF core/RIMD (Mirror1), RIMD/SMF core (Mirror2) and SMF core/surrounding medium (Mirror3), respectively.

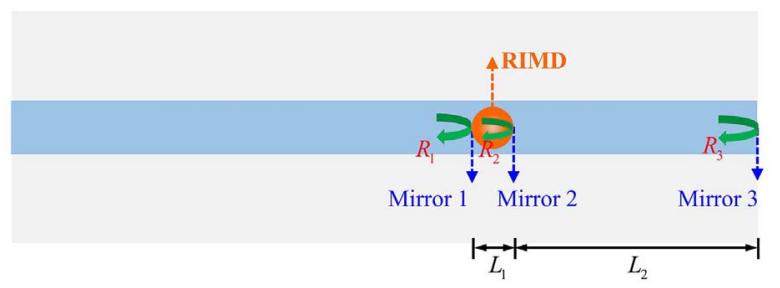

Fig. 1 Schematic diagram of the proposed composite FPI.

The light propagating in the fiber core is reflected by the three mirrors will generate a three-wave FPI pattern in fiber. For $n_{S R I}<n_{c o}$ ( $n_{S R I}$ is the RI of the surrounding medium), the normalized strength of the interference fringes can be modeled using the following three-beam optical interference equation [6]

$$
\begin{aligned}
I= & R_{1}+A^{2} R_{2}+B^{2} R_{3}-2 \sqrt{R_{1} R_{2}} A \cos \left(2 \varphi_{1}\right)- \\
& 2 \sqrt{R_{1} R_{3}} B \cos \left(2 \varphi_{1}+2 \varphi_{2}\right)+2 A B \sqrt{R_{2} R_{3}} \cos \left(2 \varphi_{2}\right)
\end{aligned}
$$

Where $A=\left(1-\alpha_{1}\right)\left(1-\gamma_{1}\right)\left(1-R_{1}\right), B=\left(1-\alpha_{1}\right)\left(1-\gamma_{1}\right)(1-$ $\left.R_{1}\right)\left(1-\alpha_{2}\right)\left(1-\gamma_{2}\right)\left(1-R_{2}\right) ; \varphi_{j}=4 \pi n_{j} L_{j} / \lambda$ is the phase shift in the cavity $j(j=1,2) ; n_{j}$ and $L_{j}$ are the refractive index and length of the cavity $j$, respectively; $\alpha_{1}, \alpha_{2}$ are the intensity attenuation factors of the Mirror 1 and Mirror 2, respectively; $\gamma_{1}, \gamma_{2}$ are defined as the transmission loss factors of the Cavity 1 and Cavity 2, 
respectively. $R_{i}$ is the intensity reflectivity of the three reflectors, $i(i=1,2,3)$, which can be calculated by: $R_{1}=R_{2}=\left[\Delta n /\left(n_{d o t}+n_{c o}\right)\right]^{2}, \quad R_{3}=\left[\left(n_{c o}-n_{S R I}\right) /\left(n_{c o}+\right.\right.$ $\left.\left.n_{S R I}\right)\right]^{2}$. Here $n_{c o}$ is the core RI of SMF, $n_{d o t}$ is the RI of the RIMD and $\Delta n=n_{d o t}-n_{c o}$. According to Eq. (1), we calculate the normalized reflection spectra from $1300 \mathrm{~nm}$ to

(a)
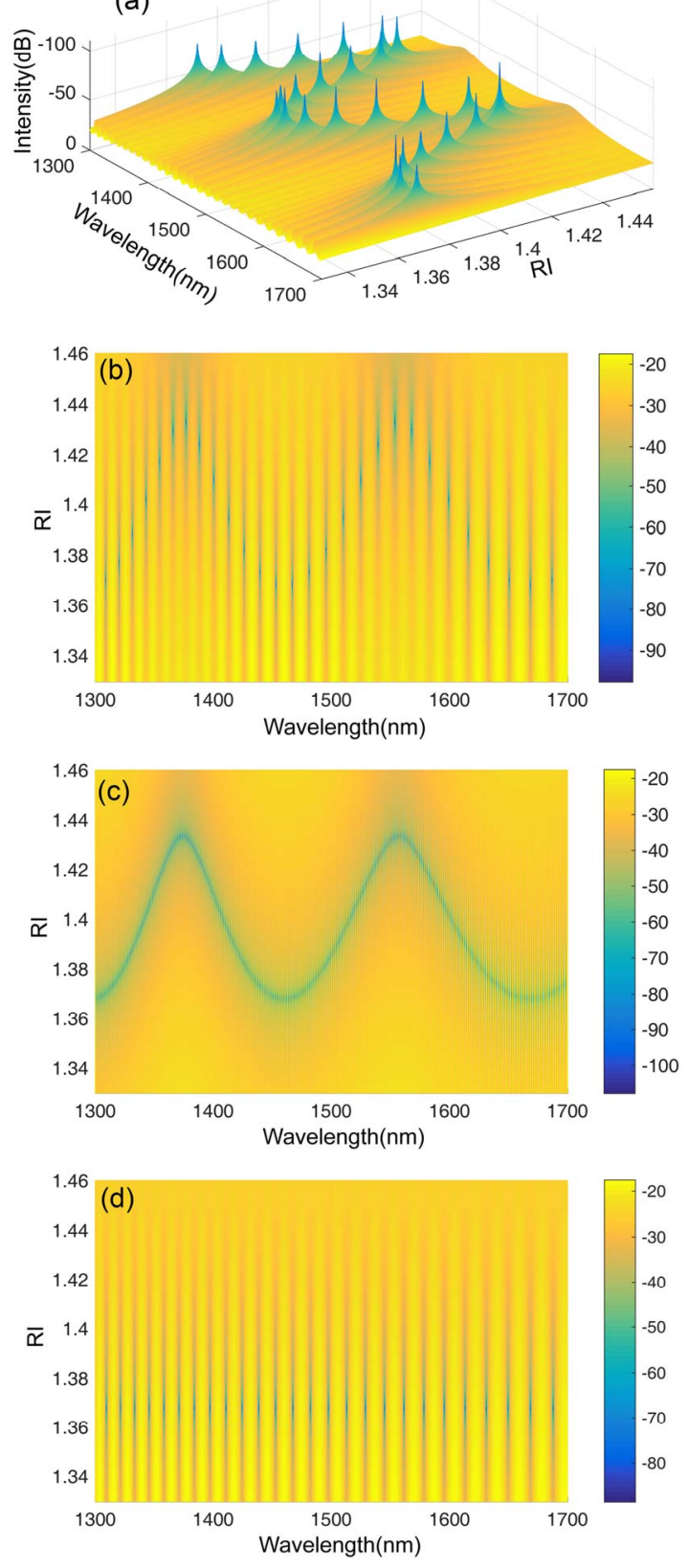

Fig. 2(a) the front and (b) top view of the composite FPI ( $L_{1}=4 \mu \mathrm{m}, L_{2}=50 \mu \mathrm{m}$ ) simulation reflection spectrum for RI change, respectively. (c) The top view of the composite FPI $\left(L_{1}=4 \mu \mathrm{m}, \quad L_{2}=500 \mu \mathrm{m}\right)$ simulation reflection spectrum for RI change. (d) The top view of the single FPI ( $L_{1}=0, L_{2}=50 \mu \mathrm{m}$ ) simulation reflection spectrum for RI change.
$1700 \mathrm{~nm}$ with parameters $\alpha_{1}=\alpha_{2}=0.3, \gamma_{1}=\gamma_{2}=0.2, \Delta n=$ $-0.018, n_{c o}=1.46, L_{1}=4 \mu \mathrm{m}, L_{2}=50 \mu \mathrm{m}$, respectively. The front and top view of calculated reflection spectra under various surrounding RIs are shown in Fig. 2(a) and (b), respectively. From which, one can clearly see that the reflection spectrum features dense fringes are modulated by a low-frequency envelope. The free spectral range (FSR) of these dense fringes corresponds to the $50 \mu \mathrm{m}$ long cavity 2 , while the low-frequency envelope stems from the $4 \mu \mathrm{m}$ short cavity 1 .

Interestingly, one can clearly see that the different interference fringe dips correspond to different ultra-sensitive zones for RI measurement (shown in blue/green on the figures). However, these ultra-sensitive zones are discrete, which is not convenient for continuous RI measurement. To reduce the spacing of these discrete ultra-sensitive zones, one can increase the length of cavity $2\left(L_{2}\right)$, which is achieved by changing the distance between the short cavity and the end of the SMF. The calculated reflection spectrum with $L_{2}=500 \mu \mathrm{m}$ is shown in Fig. 2(c), where the longer $L_{2}$ increase the number of peaks so that they become quasi-continuous. For comparison, we also calculated the reflection spectrum with $L_{1}=0$ (i.e., the device is not a composite cavity but a single cavity), as shown in Fig. 2(d). Compared Fig. 2(d) with (b), one can see that, without the envelope modulation caused by the short cavity, the ultra-sensitive regions of the respective interference dips point to the same RI value (at $\sim 1.37$ ), which means the ultra-sensitive zone is limited to a small range of measurable RI values as you travel from the minimum to maximum of the peaks. While for the composite FPI ( $\left.L_{1}=4 \mu \mathrm{m}, L_{2}=500 \mu \mathrm{m}\right)$, the ultra-sensitive zone can be widened into the range of $\sim 1.37$ to $\sim 1.43$.

\section{EXPERIMENT RESULTS}

A simple structure was adopted to verify the above theoretical model. An RIMD was induced into an end well cleaved SMF using a fs-laser. The fabrication of this device is very straightforward and the specific fabrication equipment and process are similar to our previous work [6].

We investigated the response of the composite FPI to surrounding RI using sucrose solutions with different concentrations. Fig. 3(a) shows the front view of the experimental reflection spectrum of a sample with designed $L_{2}=70 \mu \mathrm{m}$ under different RI. It can be observed clearly that different interference fringes reach the ultra-sensitive zones at different RIs, the trend of which are consistent with the theory calculations. To observe the spectral more thoroughly, Fig. 3(b) shows the typical reflection spectrum under different RIs. The FSR of the fine interference fringes is $\sim 11.6 \mathrm{~nm}$, and thus the corresponding cavity length can be calculated by $L_{2}=\lambda_{1} \lambda_{2} / 2 n_{c o} F S R=$ $66.8 \mu \mathrm{m}$ (at $1500 \mathrm{~nm}$ and the RI of the core $n_{c o}=1.46$ ), which is very close to the designed value. It indicates that $L_{2}$ can be controlled easily. The coarse fringe results from the short dot-cavity. 

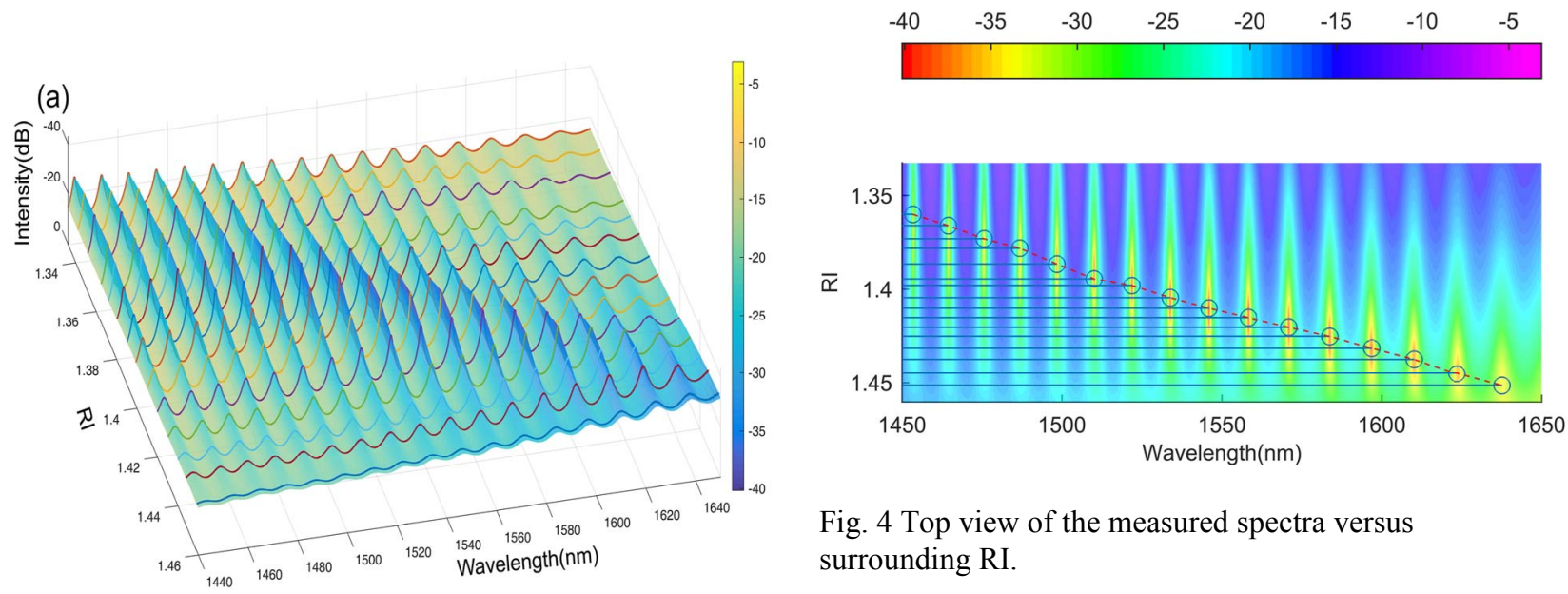

Fig. 4 Top view of the measured spectra versus surrounding RI.

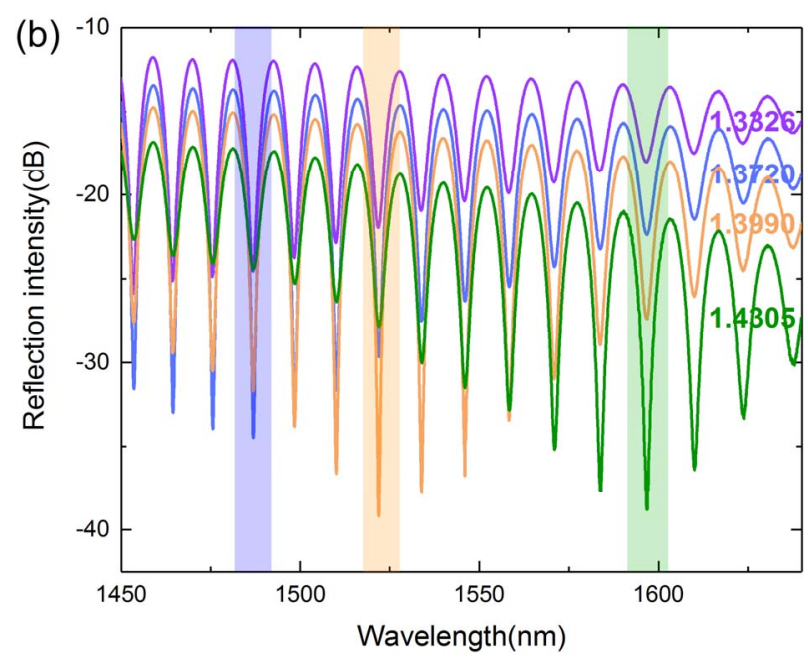

\section{CONCLUSION}

In conclusions, we discover the co-existence of high sensitivity and large dynamic range in a composite intrinsic FPI for RI sensing. To verify such a characteristic, a simple configuration is proposed, which is constituted by simply inducing a refractive-index-modified-dot in a fiber core using a fs-laser. The short RIMD cavity defines the large dynamic range, while the long SMF core cavity provides the high sensitivity ability for RI sensing. To the authors' knowledge, it is the first time that this important phenomenon in a composite FPI for RI measurement is observed. Additional merits, such as low cost, a simple and compact structure, good robust, time-saving in fabrication and the ability to overcome the temperature cross sensitivity [6] make this device a very promising candidate for practical sensing applications.

Fig.3 The front view (a) of and typical (b) measured reflective spectra of the composite FPI in different surrounding RI.

Fig. 4 shows the top view of the measured spectra versus surrounding RI. The interference fringe dip at the wavelength of $1453.4 \mathrm{~nm}$ reaches the ultra-sensitive zone at the RI of $\sim 1.359$, while the interference fringe dip at the wavelength of $1637.68 \mathrm{~nm}$ reaches at the RI of $\sim 1.452$, as shown in Fig.3(a) and Fig. 4. With the increase in wavelength of the deepest interference fringe dip at different surrounding $\mathrm{RI}$, the ultra-sensitive zones are successively reached. These results indicate that our composite FPI can achieve the co-existence of high sensitivity and larger dynamic range in RI measurement, the trends of which are in good accord with the theoretical predictions. Moreover, the red dash curve in Fig. 4 shows the recorded deepest fringe wavelength location versus RI. One can see that the location of the deepest interference fringe has an obvious "red shift" in the RI range of 1.359 to 1.452 , which is also in good accord with the theoretical calculation. Potentially, one may also use this location information as an additional indicator to decode the RI information.

\section{REFERENCES}

[1] J. Li, L. P. Sun, S. Gao, Z. Quan, Y. L. Chang, Y. Ran, L. Jin, and B. O. Guan, "Ultrasensitive refractive-index sensors based on rectangular silica microfibers," Opt. Lett. 36(18), 3593-3595 (2011).

[2] V. Bhatia and A. M. Vengsarkar, "Optical fiber long-period grating sensors," Opt. Lett. 21(9), 692-694 (1996).

[3] Y. Lai, K. Zhou, L. Zhang, and I. Bennion, "Microchannels in conventional single-mode fibers," Opt. Lett. 31(17), 2559-2561 (2006).

[4] S. Silva, O. Frazão, J. L. Santos, and F. X. Malcata, "A reflective optical fiber refractometer based on multimode interference," Sensor. . Actuat. B-Chem. 161, 88-92 (2012).

[5] Z.L. Ran, Y. J. Rao, W. J. Liu, X. Liao, and K. S. Chiang, "Laser-micromachined Fabry-Perot optical fiber tip sensor for high-resolution temperature-independent measurement of refractive index," Opt. express 16, 2252-2263 (2008).

[6] P. Chen, X. Shu, H. Cao, and K. Sugden, "Ultra-sensitive refractive index sensor based on an extremely simple femtosecond-laser-induced structure," Opt. Lett. 42(6), 1157 (2017). 